\title{
Soft Microelectrode Linear Array for Scanning Electrochemical Microscopy
}

\author{
Fernando Cortés-Salazar, ${ }^{\dagger}$ Dmitry Momotenko, ${ }^{\dagger}$ Andreas Lesch, ${ }^{\ddagger}$ Gunther Wittstock, ${ }^{\ddagger}$ and \\ Hubert H. Girault*,t
}

Laboratoire d'Electrochimie Physique et Analytique, Ecole Polytechnique Fédérale de Lausanne, Station 6, CH-1015 Lausanne, Switzerland, and Department of Pure and Applied Chemistry, Center of Interface Science (CIS), Faculty of Mathematics and Natural Sciences, Carl von Ossietzky University of Oldenburg, D-26111 Oldenburg, Germany

\begin{abstract}
A linear array of eight individual addressable microelectrodes has been developed in order to perform highthroughput scanning electrochemical microscopy (SECM) imaging of large sample areas in contact regime. Similar to previous reports, the soft microelectrode array was fabricated by ablating microchannels on a polyethylene terephthalate (PET) film and filling them with carbon ink. Improvements have been achieved by using a $5 \mu \mathrm{m}$ thick Parylene coating that allows for smaller working distances, as the probe was mounted with the Parylene coating facing the sample surface. Additionally, the application of a SECM holder allows scanning in contact regime with a tilted probe, reducing the topographic effects and assuring the probe bending direction. The main advantage of the soft microelectrode array is the considerable decrease in the experimental time needed for imaging large sample areas. Additionally, soft microelectrode arrays are very stable and can be used several times, since the electrode surface can be regenerated by blade cutting. Cyclic voltammograms and approach curves were recorded in order to assess the electrochemical properties of the device. An SECM image of a gold on glass chip was obtained with high resolution and sensitivity, proving the feasibility of soft microelectrode arrays to detect localized surface activity. Finite element method (FEM) simulations were performed in order to establish the effect of diffusion layer overlapping between neighboring electrodes on the respective approach curves.
\end{abstract}

Scanning electrochemical microscopy (SECM) is a scanning probe microscope technique that is being extensively employed for the spatial characterization of chemical reactivity at different interfaces (i.e., liquid/solid, liquid/liquid, and liquid/gas) with a high resolution and high sensitivity. ${ }^{1-4}$ Instrumentally, SECM comprises a (bi)potentiostat for recording the current generated at a microelectrode, which is horizontally $(x, y)$ and vertically $(z)$ scanned over a substrate by using a fine positioning system. For surface reactivity imaging, the microelectrode is biased at a potential where diffusion-limited electrolysis of a redox mediator

\footnotetext{
* Corresponding author. Phone: +41-21-693 3145. Fax: +41-21-693 3667. E-mail: hubert.girault@epfl.ch.

† Ecole Polytechnique Fédérale de Lausanne.

* Carl von Ossietzky University of Oldenburg.
}

occurs and the changes on the steady-state current at the scanning microelectrode are associated with local reaction sites on the specimen surface. In addition, quantitative kinetic information can be extracted, by comparing experimental data with numerical simulations of coupled heterogeneous kinetics and mass transport phenomena. ${ }^{5-12}$ As a consequence, SECM has found several applications on diverse fields ranging from the study of living cells, ${ }^{13,14}$ localized corrosion, ${ }^{15,16}$ imaging human fingerprints, ${ }^{17}$ screening electrocatalysts, ${ }^{18}$ patterning surfaces,${ }^{19}$ and investigating kinetics of very fast heterogeneous reactions. ${ }^{20,21}$

For a rather long time, instrumental developments have aimed at increasing the lateral resolution and providing complementary information such as topography and local reactivity from complex samples. For instance, combination of the SECM principle with atomic force microscopy (AFM) ${ }^{22-32}$ or electrochemical tunneling

(1) Engstrom, R. C.; Weber, M.; Wunder, D. J.; Burgess, R.; Winquist, S. Anal. Chem. 1986, 58, 844.

(2) Liu, H. Y.; Fan, F. R. F.; Lin, C. W.; Bard, A. J. J. Am. Chem. Soc. 1986, 108, 3838.

(3) Bard, A. J.; Fan, F. R. F.; Pierce, D. T.; Unwin, P. R.; Wipf, D. O.; Zhou, F. Science 1991, 254, 68.

(4) Barker, A. L.; Gonsalves, M.; MacPherson, J. V.; Slevin, C. J.; Unwin, P. R. Anal. Chim. Acta 1999, 385, 223.

(5) Kwak, J.; Bard, A. J. Anal. Chem. 1989, 61, 1221.

(6) Mirkin, M. V.; Bard, A. J. J. Electroanal. Chem. 1992, 323, 29.

(7) Wei, C.; Bard, A. J.; Mirkin, M. V. J. Phys. Chem. 1995, 99, 16033.

(8) Slevin, C. J.; Macpherson, J. V.; Unwin, P. R. J. Phys. Chem. B 1997, 101, 10851.

(9) Amphlett, J. L.; Denuault, G. J. Phys. Chem. B 1998, 102, 9946.

(10) Sklyar, O.; Wittstock, G. J. Phys. Chem. B 2002, 106, 7499.

(11) Nann, T.; Heinze, J. Electrochim. Acta 2003, 48, 3975.

(12) Cornut, R.; Lefrou, C. J. Electroanal. Chem. 2008, 621, 178.

(13) Shiku, H.; Shiraishi, T.; Aoyagi, S.; Utsumi, Y.; Matsudaira, M.; Abe, H.; Hoshi, H.; Kasai, S.; Ohya, H.; Matsue, T. Anal. Chim. Acta 2004, 522, 51.

(14) Yasukawa, T.; Kaya, T.; Matsue, T. Electroanalysis 2000, 12, 653.

(15) Fushimi, K.; Okawa, T.; Azumi, K.; Seo, M. J. Electrochem. Soc. 2000, 147, 524.

(16) Fushimi, K.; Seo, M. Zairyo to Kankyo 1997, 46, 797.

(17) Zhang, M.; Girault, H. H. Analyst 2009, 134, 25.

(18) Walsh, D. A.; Fernandez, J. L.; Bard, A. J. J. Electrochem. Soc. 2006, 153, E99.

(19) Mandler, D. In Scanning Electrochemical Microscopy; Bard, A. J., Mirkin, M. V., Eds.; Marcel Dekker: New York, 2001; p 593.

(20) Sun, P.; Mirkin, M. V. Anal. Chem. 2006, 78, 6526.

(21) Velmurugan, J.; Sun, P.; Mirkin, M. V. J. Phys. Chem. C 2009, 113, 459.

(22) Macpherson, J. V.; Unwin, P. R. Anal. Chem. 2000, 72, 276.

(23) Kranz, C.; Friedbacher, G.; Mizaikofft, B.; Lugstein, A.; Smoliner, J.; Bertagnolli, E. Anal. Chem. 2001, 73, 2491.

(24) Kueng, A.; Kranz, C.; Lugstein, A.; Bertagnolli, E.; Mizaikoff, B. Angew. Chem., Int. Ed. 2003, 42, 3238. 
microscopy (EC-STM) ${ }^{33}$ have in particular addressed the question of keeping a constant working distance $d$ over corrugated samples. Another approach to compensate the sample tilt is the use of multiple probes (e.g., double barrels, heptodes, dual microelectrodes) in which one is used for determination of $d$ and the other to follow the reaction at the substrate. ${ }^{34-36}$ Multiple probes can also be used for enhancing image information or be used sequentially. ${ }^{37-40}$ Nonelectrochemical distance information can be obtained by shear-force measurements. ${ }^{41-46}$ The fastest imaging can be obtained by the contact principle because it avoids double scanning (such as in lift-off) and does not need to consider the finite time constant of an electronic feedback loop that keeps the distance constant based on an electrochemical or shear-force signal.

Recently, other SECM applications such as screening combinatorial catalyst libraries, ${ }^{47-50}$ assessing the integrity of coatings, ${ }^{15,16,51}$ reading-out electropherograms, ${ }^{52}$ human fingerprints $^{17}$ or protein arrays ${ }^{53,54}$ call for larger scanning areas approaching the square centimeter. With current instrumentation,

(25) Sklyar, O.; Kueng, A.; Kranz, C.; Mizaikoff, B.; Lugstein, A.; Bertagnolli, E.; Wittstock, G. Anal. Chem. 2005, 77, 764.

(26) Davoodi, A.; Pan, J.; Leygraf, C.; Norgren, S. Electrochim. Acta 2007, 52, 7697.

(27) Fasching, R. J.; Tao, Y.; Prinz, F. B. In Proceedings of SPIE-International Society for Optical Engineering; Maher, M. A., Jakubczak, J. F., Eds.; SPIE: San Jose, CA, 2004; Vol. 5342, p 53.

(28) Frederix, P. L. T. M.; Bosshart, P. D.; Akiyama, T.; Chami, M.; Gullo, M. R.; Blackstock, J. J.; Dooleweerdt, K.; De Rooij, N. F.; Staufer, U.; Engel, A. Nanotechnology 2008, 19, 384004.

(29) Ueda, A.; Niwa, O.; Maruyama, K.; Shindo, Y.; Oka, K.; Suzuki, K. Angew. Chem., Int. Ed. 2007, 46, 8238.

(30) Davoodi, A.; Farzadi, A.; Pan, J.; Leygraf, C.; Zhu, Y. J. Electrochem. Soc. 2008, 155, C474.

(31) Dobson, P. S.; Weaver, J. M. R.; Holder, M. N.; Unwin, P. R.; Macpherson, J. V. Anal. Chem. 2005, 77, 424.

(32) Sklyar, O.; Treutler, T. H.; Vlachopoulos, N.; Wittstock, G. Surf. Sci. 2005, $597,181$.

(33) Treutler, T. H.; Wittstock, G. Electrochim. Acta 2003, 48, 2923.

(34) Baur, J. E.; Miller, H. M.; Ritchason, M. A. Anal. Chim. Acta 1999, 397, 123.

(35) Wei, C.; Bard, A. J.; Nagy, G.; Toth, K. Anal. Chem. 1995, 67, 1346.

(36) Yasukawa, T.; Kaya, T.; Matsue, T. Anal. Chem. 1999, 71, 4637.

(37) Liljeroth, P.; Johans, C.; Slevin, C. J.; Quinn, B. M.; Kontturi, K. Anal. Chem. 2002, 74, 1972.

(38) Ufheil, J.; Borgwarth, K.; Heinze, J. Anal. Chem. 2002, 74, 1316.

(39) Sklyar, O.; Ufheil, J.; Heinze, J.; Wittstock, G. Electrochim. Acta 2003, 49, 117.

(40) Isik, S.; Etienne, M.; Oni, J.; Blachi, A.; Reiter, S.; Schuhmann, W. Anal. Chem. 2004, 76, 6389.

(41) Ludwig, M.; Kranz, C.; Schuhmann, W.; Gaub, H. E. Rev. Sci. Instrum. 1995, 66, 2857.

(42) James, P. I.; Garfias-Mesias, L. F.; Moyer, P. J.; Smyrl, W. H. J. Electrochem. Soc. 1998, 145, L64.

(43) Lee, Y.; Bard, A. J. Anal. Chem. 2002, 74, 3626.

(44) Ballesteros Katemann, B.; Schulte, A.; Schuhmann, W. Chem.-Eur. J. 2003, 9, 2025.

(45) Oyamatsu, D.; Hirano, Y.; Kanaya, N.; Mase, Y.; Nishizawa, M.; Matsue, T. Bioelectrochemistry 2003, 60, 115

(46) Garay, M. F.; Ufheil, J.; Borgwarth, K.; Heinze, J. Phys. Chem. Chem. Phys. 2004, 6, 4028.

(47) Fernandez, J. L.; Walsh, D. A.; Bard, A. J. J. Am. Chem. Soc. 2005, 127, 357.

(48) Black, M.; Cooper, J.; McGinn, P. Meas. Sci. Technol. 2005, 16, 174.

(49) Minguzzi, A.; Alpuche-Aviles, M. A.; Lopez, J. R.; Rondinini, S.; Bard, A. J. Anal. Chem. 2008, 80, 4055.

(50) Bard, A. J. J. Am. Chem. Soc. 2010, 132, 7559.

(51) Rianasari, I.; Walder, L.; Burchardt, M.; Zawisza, I.; Wittstock, G. Langmuir 2008, 24, 9110 .

(52) Zhang, M.; Wittstock, G.; Shao, Y.; Girault, H. H. Anal. Chem. 2007, 79, 4833.

(53) Wittstock, G. Fresenius J. Anal. Chem. 2001, 370, 303. this poses a problem because the sample tilt must be minimized. While this might be possible for an experienced operator, curved samples are very difficult to deal with and imaging times become so long that artifacts arising from electrode fouling, supporting electrolyte evaporation, or sample aging are introduced.

Usually three different strategies are followed to overcome such problems. The first strategy is based on the increment of the scanning rate and step size of the probe, in which case the image resolution is compromised. Scan rates up to $500 \mu \mathrm{m} \cdot \mathrm{s}^{-1}$ and step sizes of $150 \mu \mathrm{m}$ have been employed with this aim. ${ }^{48,55}$ However, Combellas et al. showed that contribution to the measured current originated from convection cannot be neglected when scanning at rates higher than $10 \mu \mathrm{m} \cdot \mathrm{s}^{-1}{ }^{56}$ Indeed, at extreme fast scan rates $\left(\sim 1000 \mu \mathrm{m} \cdot \mathrm{s}^{-1}\right)$ the capability for detecting surface reactivity is lost. $^{56}$ A second strategy employed to reduce problems arising from evaporation and electrode fouling is to image subregions of a large sample area with renewal of supporting electrolyte and/or electrode surface in between individual image frames. ${ }^{48,57}$ For instance, areas close to $2 \mathrm{~cm}^{2}$ have been examined by SECM in order to screen combinatorial libraries for fuel cell electrode applications. ${ }^{48}$ The third strategy is based on using multiple independent electrodes. Consequently, a large area is scanned in less time without compromising the image quality and avoiding the associated experimental problems outlined above. Such an approach has been largely followed in AFM imaging. ${ }^{58-60}$

Already in 1995, Meyer et al. had reported imaging experiments in the generation-collection mode using 400 individually addressable microelectrodes. ${ }^{61}$ The imaging depended completely on the layout of the two-dimensional electrode array, and no displacement of the multiprobe was employed. Parallel arrays have been used in a SECM-like configuration in the context of the confined etchant layer technique. ${ }^{62}$ Here the problem of diffusional interference at 2D array of electrode was overcome by a homogeneous scavenger reaction and periodically lifting the tool electrode to renew the solution in the interelectrode gap. Recently, a multielectrode probe for parallel SECM imaging has been presented by Barker et al. that comprised a linear array of 16 independent, slightly recessed Pt $10 \mu$ m-diameter microelectrodes on a planar insulating chip. ${ }^{63}$ For scanning purposes, the bundle was kept in a fixed position while the sample was moved along the $x$ and $y$ axes. The array cannot be regenerated by mechanical polishing because this would not only destroy the $200 \mathrm{~nm}$ thick nitride film that protects the leads of each individual electrode,

(54) Nunes Kirchner, C.; Traeuble, M.; Wittstock, G. Anal. Chem. 2010, 82, 2626.

(55) Liu, W.; Ye, H.; Bard, A. J. J. Phys. Chem. C 2010, 114, 1201.

(56) Combellas, C.; Fermigier, M.; Fuchs, A.; Kanoufi, F. Anal. Chem. 2005, $77,7966$.

(57) Zoski, C. G.; Simjee, N.; Guenat, O.; Koudelka-Hep, M. Anal. Chem. 2004, 76,62 .

(58) Hong, S.; Mirkin, C. A. Science 2000, 288, 1808.

(59) Vettiger, P.; Despont, M.; Drechsler, U.; Dorig, U.; Haberle, W.; Lutwyche, M. I.; Rothuizen, H. E.; Stutz, R.; Widmer, R.; Binnig, G. K. IBM J. Res. Dev. 2000, 44, 323.

(60) Fasching, R. J.; Tao, Y.; Prinz, F. B. Sens. Actuators, B 2005, 108, 964.

(61) Meyer, H.; Drewer, H.; Gründig, B.; Cammann, K.; Kakerow, R.; Manoli, Y.; Mokwa, W.; Rospert, M. Anal. Chem. 1995, 67, 1164.

(62) Zhang, L.; Ma, X. Z.; Zhuang, J. L.; Qiu, C. K.; Du, C. L.; Tang, J.; Tian, Z. W. Adv. Mater. 2007, 19, 3912.

(63) Barker, A. L.; Unwin, P. R.; Gardner, J. W.; Rieley, H. Electrochem. Commun. 2004, 6, 91 . 
but also the $130 \mathrm{~nm}$ thick Pt film. ${ }^{63}$ Leïchle et al. used an electrically contacted cantilever with integrated microfluidics for localized copper deposition. ${ }^{64}$

An important issue when working with arrays is the crosstalk between electrodes, which could be avoided if the thickness of the diffusion layer of each microelectrode is smaller than half the distance between adjacent electrodes. ${ }^{65}$ With this aim, Barker et al. employed an interelectrode spacing equal to $120 \mu \mathrm{m}$ for a $10 \mu$ m-diameter microelectrode that should be sufficient for avoiding diffusion layer overlapping. However, lower currents than expected were observed by cyclic voltammetry that could indicate an overlap of the diffusion layers of neighboring electrodes. The latter is most likely due to the fact the diffusional independence of microelectrodes in arrays is only valid for a finite time scale that depends on the distance between neighboring probes. ${ }^{66-71}$

In the present article, we introduce a new concept using an array of individually addressable microelectrodes for SECM imaging based on the recent development of the microfabricated soft stylus probes. ${ }^{72}$ Additionally, improvements related to the scanning in contact mode with an inclined probe coated by a thin Parylene film have been introduced. The soft nature of the electrode allows imaging while the probe is in mechanical contact with the sample. This defines a constant working distance for the electrodes sandwiched between two sheets of insulators similar to the SECM-AFM imaging where the working distance is defined by height of the AFM tip protruding from a sheath containing the microelectrode. ${ }^{23}$ By exposing the microelectrode array on the cross-section of a sheet structure, rather than on the surface of a chip-like overall shape, the problems of diffusional shielding are reduced and the contacting of the array elements is greatly simplified. Finite element method (FEM) simulations were performed in order to establish the effect of diffusion layer overlapping between neighboring electrodes on the respective approach curves. As a proof of concept for high-resolution imaging of large samples, a gold electrode structure on a glass chip was imaged by a soft microelectrode array.

\section{EXPERIMENTAL SECTION}

Chemicals. Ferrocene methanol $\left(\mathrm{FcCH}_{2} \mathrm{OH}, \geq 97 \%\right)$, bovine serum albumin (BSA) ( $\geq 90 \%$ ), benzoquinone ( $\geq 99.5 \%)$, and $\mathrm{KCl}$ and $\mathrm{KNO}_{3}(\geq 99 \%)$ were purchased from Sigma-Aldrich (Schnelldorf, Switzerland). Methanol and $\mathrm{K}_{3}\left[\mathrm{Fe}(\mathrm{CN})_{6}\right](\geq 99 \%)$ were purchased from Merck (Dietikon, Switzerland). All the chemicals were used as received. Deionized water was produced by a Milli-Q plus 185 model from Millipore (Zug, Switzerland).

(64) Leïchlé, T.; Nicu, L.; Descamps, E.; Corso, B.; Mailley, P.; Livache, T.; Bergaud, C. Appl. Phys. Lett. 2006, 88, 254108.

(65) Stulik, K.; Amatore, C.; Holub, K.; Marecek, V.; Kutner, W. Pure Appl. Chem. 2000, 72, 1483 .

(66) Amatore, C.; Saveant, J. M.; Tessier, D. J. Electroanal. Chem. 1983, 147, 39.

(67) Shoup, D.; Szabo, A. J. Electroanal. Chem. 1984, 160, 19.

(68) Menshykau, D.; Huang, X.; Rees, N. V.; del Campo, F. J.; Munoz, F. X.; Compton, R. G. Analyst 2009, 134, 343.

(69) Wittstock, G.; Grondig, B.; Strehlitz, B.; Zimmer, K. Electroanalysis 1998 , 10,526 .

(70) Bond, A. M.; Oldham, K. B.; Zoski, C. G. Anal. Chim. Acta 1989, 216, 177.

(71) Wightman, R. M.; Wipf, D. O. In Electroanalytical Chemistry; Bard, A. J., Ed.; Marcel Dekker: New York, 1989; Vol. 15, p 267.

(72) Cortes-Salazar, F.; Traeuble, M.; Li, F.; Busnel, J.-M.; Gassner, A.; Hojeij, M.; Wittstock, G.; Girault, H. H. Anal. Chem. 2009, 81, 6889.
Preparation of Gold Electrodes. Glass slides were treated with piranha solution and cleaned by sequential sonication in ethanol, acetone, and purified water followed by drying under a stream of argon. Caution: This mixture reacts violently with all organic material. Piranha solution has to be handled with extreme care to avoid personnel injury and property damage. Gold films of $100 \mathrm{~nm}$ thickness were prepared in an Edwards Auto 306 evaporator operating at a pressure less than $5 \times 10^{-6} \mathrm{mbar}$. The film growth was initiated by the thermal evaporation of a $1 \mathrm{~nm}$ thick chromium (99.99\%, Balzers) layer at $<0.1 \mathrm{~nm} / \mathrm{s}$ as adhesion promoter. Gold (99.99\%, Balzers) was subsequently evaporated at $<0.1 \mathrm{~nm} / \mathrm{s}$ up to $5 \mathrm{~nm}$, before increasing the deposition rate to $0.2-0.3 \mathrm{~nm} / \mathrm{s}$ for the deposition of a $100 \mathrm{~nm}$ layer.

Soft Microelectrodes Array Preparation. Eight microchannels were prepared into a $100 \mu \mathrm{m}$ thick polyethylene terephthalate (PET, Melinex Dupont, Wilmington, DE) film by laser ablation through a metallic mask using a $193 \mathrm{~nm}$ ArF excimer laser beam (Lambda Physik, Göttingen, Germany, fluence $=0.2 \mathrm{~J}$, frequency $=50 \mathrm{~Hz}$ ) as reported in previous papers. ${ }^{72-75}$ The average width, depth, and length of the prepared microchannels were $20 \mu \mathrm{m}, 30$ $\mu \mathrm{m}$, and $5 \mathrm{~cm}$. The electrode-to-electrode distances employed for the prepared arrays were equal to 130,250 , or $500 \mu \mathrm{m}$. Lasermachined tracks in PET were filled manually with a spatula by Electrador carbon ink (Electra Polymer \& Chemicals Ltd., Roughway Mill, Dunk Green, England) to create conductive tracks for the working electrodes. After curing at $80{ }^{\circ} \mathrm{C}$ for $1 \mathrm{~h}$, a $2-5$ $\mu \mathrm{m}$ thick Parylene $\mathrm{C}$ film was deposited over the electrodes by using a Parylene deposition system (Comelec SA, La Chaux-deFonds, Switzerland). A cross-section of the microchannels was then exposed by laser ablation, cutting with a surgical scalpel (No. 10 made in sterile stainless steel, Swan-Morton, Sheffield, England) or mechanical polishing by using a custom-built polishing machine with successively finer diamond lapping discs of different particles sizes 30, 6, and $0.1 \mu \mathrm{m}$ (Ultraprep, Buehler, Schlieren, Switzerland). The quality of the electrodes was checked with a Laborlux D optical microscope (Leitz, Germany) and a scanning laser microscope (VK 8700, Keyence).

SECM Measurements. SECM measurements were carried out using a custom-built SECM setup running under SECMx software $^{76}$ and comprising an IVIUM compactstat (IVIUM Technologies, The Netherlands) operating in a three-electrode mode. The potentiostat was complemented by the WE32 unit (IVIUM Technologies, The Netherlands) that allowed the operation of 32 individually addressable working electrodes against a common counter (Pt wire) and reference electrode (Ag wire as quasireference electrode, QRE). All potentials are reported with respect to the Ag-QRE. The interdigitated gold electrode array to be imaged was used as an unbiased substrate. All the samples were mounted on the bottom of a flat cell construction and investigated at room temperature $\left(20 \pm 2{ }^{\circ} \mathrm{C}\right)$.

(73) Roberts, M. A.; Rossier, J. S.; Bercier, P.; Girault, H. H. Anal. Chem. 1997, 69, 2035.

(74) Rossier, J. S.; Roberts, M. A.; Ferrigno, R.; Girault, H. H. Anal. Chem. 1999, $71,4294$.

(75) Cortés-Salazar, F.; Lesch, A.; Momotenko, D.; Busnel, J.-M.; Wittstock, G.; Girault, H. H. Anal. Methods 2010, 2, 817.

(76) Nunes, K.; Hallmeier, K.; Szargan, R.; Raschke, T.; Radehaus, C.; Wittstock, G. Electroanalysis 2007, 19, 1023. 
For using the new probe in contact regime, the probe was mounted with a predefined angle of $70^{\circ}$ with respect to the sample surface using a custom-built SECM holder. As a result, both probe bending degree and direction were preset before scanning in contact regime (see Figure SI-1 in Supporting Information SI-1). In order to achieve small working distances, the probe was mounted such that the $5 \mu \mathrm{m}$ thick Parylene coating faced the sample surface. For discussing the image routine, we distinguish the high-frequency axis, i.e., the horizontal direction in which individual line scans are performed. The term "high-frequency" refers to the fact that the forth and back movement of the probe along this dimension is faster than in the other lateral dimension (i.e., the low-frequency direction) in which an incremental movement is performed after each forward and reverse line scan along the high-frequency axis. A new routine was included in the SECMx software that allows the dragging of the inclined probe in the forward line scan of the high-frequency axis but the lifting of the probe in the reverse scan by a preset stroke height so that the probe is freely suspended. After returning to the start position along the high-frequency axis, the probe is moved horizontally along the low-frequency axis. The probe is brought again into mechanical contact with the sample by a vertical translation equal to the negative value of the stroke height, and the next line scan in the contact mode commences. Thus, the probe bending degree and bending direction are kept constant within one image frame when scanning in contact regime. Images are constructed from the data acquisition of forward line scans in contact mode using MIRA software. ${ }^{77}$

Simulations. Simulations with the microelectrode arrays were performed with the software package COMSOL multiphysics (version 3.5a), which employs the finite element method (FEM) for the numerical resolution of differential equations. All the simulations were performed on a Linux Ubuntu 8.04 platform with a four Core $2.66 \mathrm{GHz}$ CPU and $7.8 \mathrm{~GB}$ of RAM.

\section{RESULTS AND DISCUSSION}

Probe Characterization. A top view of the soft microelectrode array (Figure 1a) shows eight carbon tracks with an electrodeto-electrode distance equal to $500 \mu \mathrm{m}$. Each electrode leads to a contact pad located in the left part of Figure 1a that allows the electrodes to be addressed individually. Before use, a cross-section of the covered carbon tracks were exposed by laser ablation or blade cutting followed in some cases by mechanical polishing with fine diamond lapping discs. A cross-section defines an array of eight carbon microelectrodes and is presented in Figure $1 \mathrm{~b}$ (130 $\mu$ m electrode-to-electrode distance), where the successful filling and closing of the microchannel can be seen. Cutting was performed by laser ablation. The need for a UV-laser to obtain a fresh surface limits its applicability in daily laboratory routines. Figure 1c shows the cross-section of an array of eight microelectrodes (500 $\mu$ m electrode-to-electrode distance) obtained by cutting with a surgical scalpel. Although the surface is not as smooth as the laser-ablated surfaces, this process does not affect the active electrode area or the Parylene coating.

The electrochemical behavior of each microelectrode in the arrays was tested individually by cyclic voltammetry in $2 \mathrm{mM}$

(77) Wittstock, G.; Asmus, T.; Wilhelm, T. Fresenius J. Anal. Chem. 2000, 367, 346.

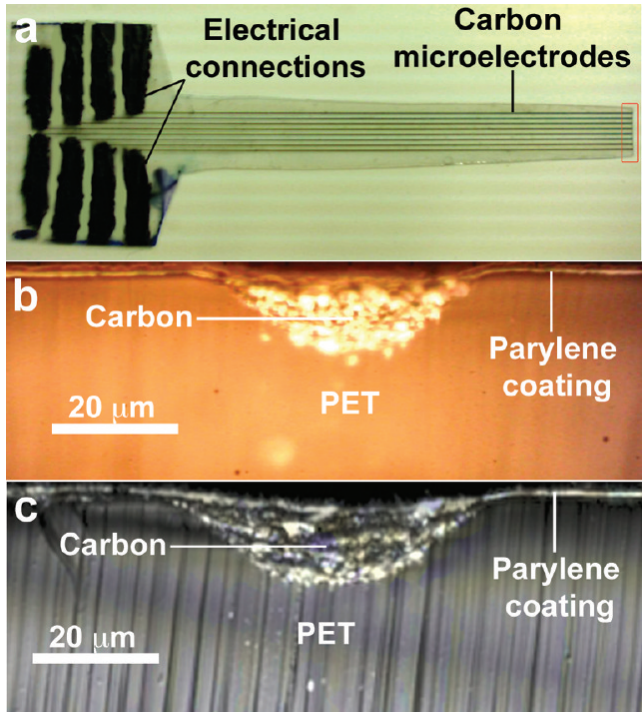

Figure 1. (a) Optical image of a soft microelectrode array (500 $\mu \mathrm{m}$ electrode spacing) employed as working electrode for SECM experiments and cross-sections of an individual carbon microelectrode exposed by (b) laser ablation (130 $\mu \mathrm{m}$ electrode spacing), and (c) cutting with a surgical scalpel blade (500 $\mu \mathrm{m}$ electrode spacing).
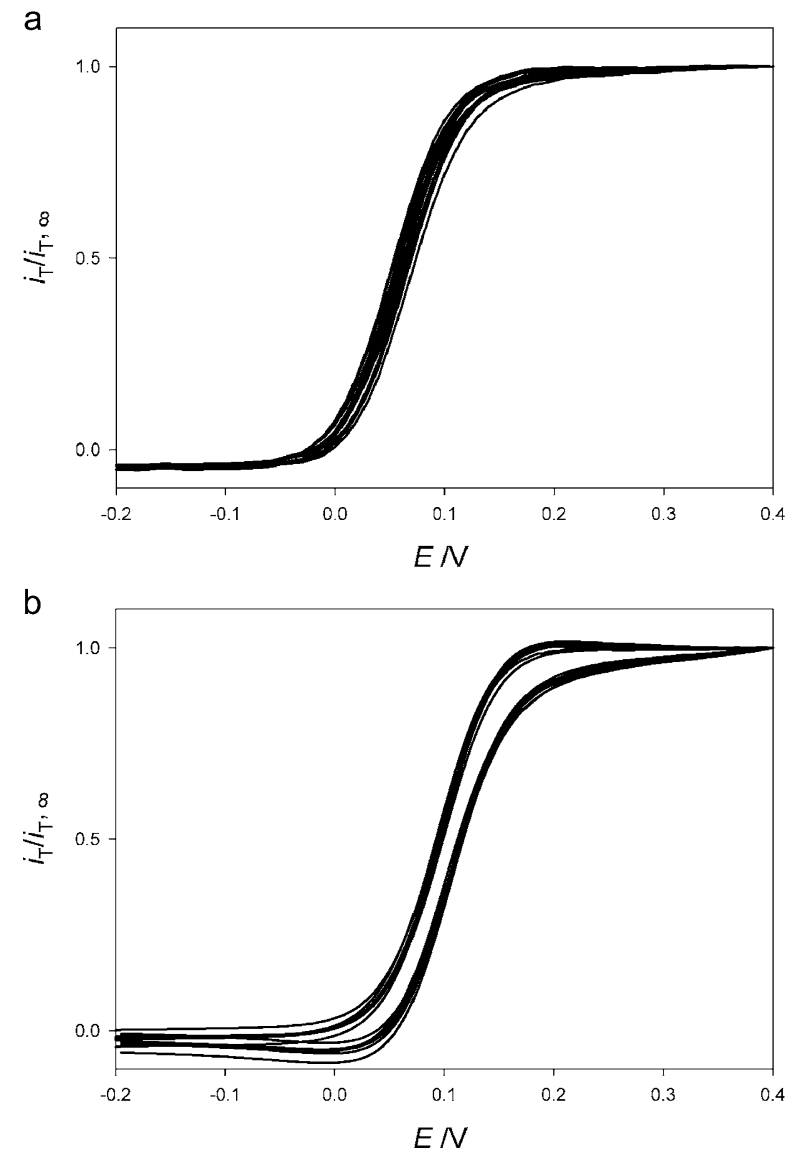

Figure 2. Normalized cyclic voltammograms of an array of eight microelectrodes exposed by (a) laser ablation and (b) cutting with surgical scalpel blade in $2 \mathrm{mM} \mathrm{FcCH}_{2} \mathrm{OH}$ and $0.1 \mathrm{M} \mathrm{KNO}_{3}$. Each microelectrode was tested individually. Scan rate $20 \mathrm{mV} / \mathrm{s}$.

$\mathrm{FcCH}_{2} \mathrm{OH}$ (Figure 2). All the cyclic voltammetries obtained were normalized by the respective steady-state current recorded at the 
solution bulk $\left(i_{\mathrm{T}, \infty}\right)$. As reported in a previous communication, ${ }^{72}$ cutting by laser ablation produces very smooth PET surfaces along with highly active carbon electrodes (Figure 2a). Carbon microelectrodes exposed by blade cutting also presented a sigmoidal response, but with higher capacitive currents (Figure $2 b)$. Nevertheless, the absolute steady-state currents recorded with the multiprobe exposed by cutting are similar compared to the ones obtained after laser ablation. Due to the microchannel filling processes, different active electrode areas in the array are obtained, and as a consequence different diffusion-limited steadystate currents are recorded. For instance, the average of the absolute steady-state current for the array exposed by blade cutting (Figure $2 \mathrm{~b}$ ) was equal to $18.3 \mathrm{nA}$ with a standard deviation of $0.8 \mathrm{nA}$. However, as observed in Figure 2, the diffusion-limited currents can be corrected by introducing a scale factor that normalizes all the current values of each individual electrode of the array (vide infra). The reproducibility on surface finishing obtained by blade cutting has been found to be adequate for the present imaging goals as shown later in Figure 5.

In comparison to the recently developed soft stylus probes, ${ }^{72}$ two improvements are introduced here for the fabrication of the array. The first one is related to the SECM holder that allows the bundle to be inclined by a predefined angle $(\beta)$ (Supporting Information SI-1). By operating the probe at an angle (see Figure SI-1a in Supporting Information SI-1) it is possible to control the degree and direction of the probe bending, and to reduce topographic artifacts coming from 3D patterns with heights higher than $100 \mu \mathrm{m}$. The angle $\beta$ decreases the contrast in $i_{\mathrm{T}}$ (i.e., current difference between active and nonactive regions) because at larger $\beta$, the mediator diffusion from the solution bulk is less effectively shielded by the inclined probe. In addition, a larger $d$ is established (see Figure SI-1b in Supporting Information SI1). A $\beta$ angle of $70^{\circ}$ was determined to be a good compromise between diminishing topographic artifacts and maintaining current contrast (results not shown). The second improvement concerns the thin Parylene coating that replaces the previously used PE/PET lamination film. This thin film coating technique provides a $5 \mu \mathrm{m}$ thick pinhole-free insulation and reduces the distance $d$ defined as the distance from the center of the active electrode area to the outer edge of the probe and therefore guarantees that the electrode is in a very close proximity to the substrate (see Figure SI-2 in Supporting Information SI-2).

Simulation of Approach Curves. FEM simulations of two microelectrode arrays with different interelectrode distances (i.e., 130 and $500 \mu \mathrm{m}$ ) were performed in order to establish the effect of the diffusion layer overlapping on the respective approach curves. An array of three microelectrodes was chosen for the simulations (Figure 3a), because it is the minimum number of microelectrodes that describes the effect on inner and outer electrodes in a linear array. A probe slope equal to $70^{\circ}$, as well as a quarter-moon shape, was assumed as the electrode geometry (i.e., $40 \mu \mathrm{m}$ width and $20 \mu \mathrm{m}$ depth) for the simulations. In order to plot the approach curves after touching the sample, a new vertical coordinate $h_{\mathrm{p}}$ was defined. It is the difference $h_{\mathrm{P}}=h_{\mathrm{A}}-l_{\mathrm{T}}$ between the height of the attachment point above the sample $h_{\mathrm{A}}$ and the vertical
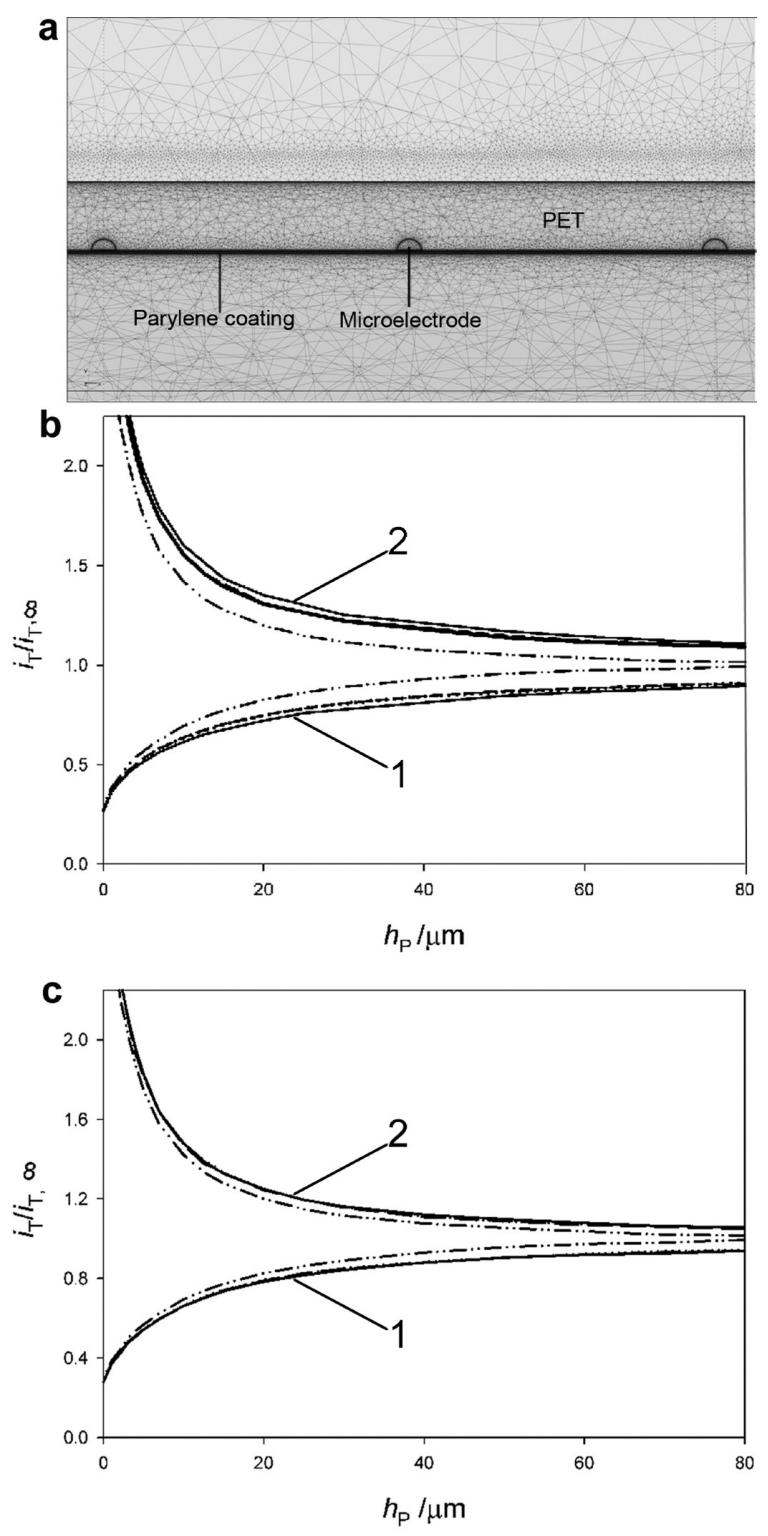

Figure 3. (a) Shape and dimension of the active electrode area considered for the FEM simulations. Simulated SECM feedback approach curves for an array with an interelectrode distance equal to (b) $130 \mu \mathrm{m}$ or (c) $500 \mu \mathrm{m}$ over an insulating sample (1) and a conductive surface with mass transport-controlled mediator regeneration (2). Outer electrodes are depicted by dashed and dotted lines, while a continuous line represents the inner electrode. The dasheddotted line corresponds to the calculated approach curve of a single microelectrode.

extension $l_{\mathrm{T}}$ of the unbent probe. Thus, the effective working distance $d$ in the contact mode is defined by

$$
d=\left\{\begin{array}{l}
d=h_{\mathrm{P}}+t_{\mathrm{L}} \sin (\alpha) ;\left(h_{\mathrm{P}} \geq 0, \text { noncontact regime }\right) \\
d=t_{\mathrm{L}} \sin (\alpha) ;\left(h_{\mathrm{P}}<0, \text { contact regime }\right)
\end{array}\right.
$$

where $\alpha$ is the angle between the cross-sectional plane of the probe and the sample surface and $t_{\mathrm{L}}$ is the thickness of the polymer film covering the carbon tracks. The geometry of the probes in contact and noncontact regime is shown in Figure SI-3 in Supporting Information SI-3. 
Approach curves were simulated only for $h_{\mathrm{P}}>0$ because elastic deformation and sliding of the probe take place when the soft probe is pressed against the substrate that cannot be described by the current simulation setup. ${ }^{72}$ Further details about simulations are available in Supporting Information SI4. Figure $3 \mathrm{~b}$ shows the simulated approach curves of an array of three microelectrodes with an interelectrode distance of $130 \mu \mathrm{m}$. The simulations predict both positive and negative feedback responses. The deviation between the behavior of an individual electrode (dashed-dotted lines in Figure 3b) and electrodes within an array results from the diffusion layer overlapping of neighboring electrodes. The overlapping on the diffusion layers decreases the currents in the case of negative feedback, as a result of the redox mediator overconsumption produced by the neighboring electrodes. For positive feedback, a higher current is detected because each electrode also benefits from electrolysis at neighboring electrodes. Similar results have been obtained when working with heptode microelectrodes in disk generation-ring generation (DG-RG) mode, where overlapping of the diffusion layers among electrodes increased and decreased positive and negative feedback, respectively. ${ }^{39}$

It is important to notice that electrode cross-talk effect is more relevant for inner electrodes (continuous lines), but also the response of outer electrodes deviates (dashed and dotted lines) from the response of a single probe (dashed-dotted lines). According to Figure 3b, the highest difference in current-distance curves between outer and inner electrodes is 2.6\%. This should allow the use of these electrodes for qualitative SECM imaging in the feedback mode. Figure 3c shows the simulated approach curves performed with an array of three electrodes with an electrode-to-electrode separation of $500 \mu \mathrm{m}$. In contrast to the array with an electrode spacing equal to $130 \mu \mathrm{m}$, no clear difference is observed for the approach curves obtained for outer and inner electrodes. Moreover, in comparison to the approach curves calculated for a single microelectrode the highest difference corresponds to $2.1 \%$ when the probes are close to the substrate. According to Figure 3c, a small diffusion layer overlapping takes place when using an array of microelectrodes with an electrode spacing $\geq 500 \mu \mathrm{m}$. It has to be pointed out that when approaching the substrate either for positive or negative feedback, the differences among all the electrodes diminishes, probably as a result of the compression of the diffusion layer.

Approach Curve Characterization. Experimental approach curves over insulating (glass) and conducting (gold film) substrates were performed in $2 \mathrm{mM} \mathrm{FcCH}_{2} \mathrm{OH}$ (Figure 4). The oxidation of $\mathrm{FcCH}_{2} \mathrm{OH}$ under diffusion-controlled conditions at all the microelectrodes was achieved by biasing the array at $E_{\mathrm{T}}=0.3 \mathrm{~V}$ during all the experiments. As the array of microelectrodes was approached toward an insulating sample, a decrease of the current was recorded due to the hindered $\mathrm{FcCH}_{2} \mathrm{OH}$ diffusion (negative feedback). In contrast, when the microelectrode array was brought into a close contact with a conductive region, an increase of the current was observed, as recycling of the redox mediator was achieved by the $\mathrm{FcCH}_{2} \mathrm{OH}^{+}$reduction at the unbiased gold film (positive feedback). The driving force for the regeneration of $\mathrm{FcCH}_{2} \mathrm{OH}$ results from a concentration cell formed by the deviation of the $\left[\mathrm{FeCH}_{2} \mathrm{OH}\right] /\left[\mathrm{FeCH}_{2} \mathrm{OH}^{+}\right]$under the microelectrodes from

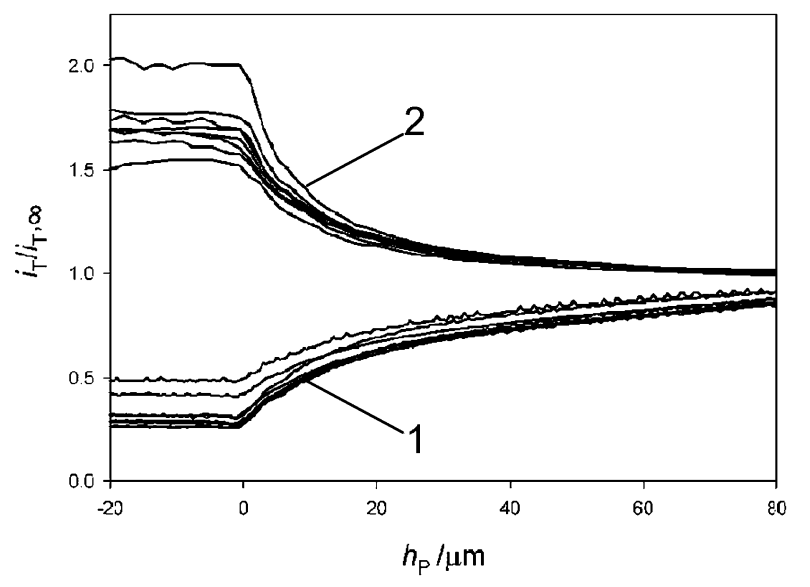

Figure 4. Experimental approach curves with an array of eight microelectrodes ( $130 \mu \mathrm{m}$ interelectrode spacing) over insulating glass (1) and unbiased gold electrode (2), in $2 \mathrm{mM} \mathrm{FcCH} \mathrm{FH}_{2} \mathrm{OH}$ and $0.1 \mathrm{M}$ $\mathrm{KNO}_{3} . E_{\mathrm{T}}=0.35 \mathrm{~V}$, translation rate $0.5 \mu \mathrm{m} / \mathrm{s}$.

the corresponding values far away from the probe. ${ }^{78}$ As a consequence, recycling of the redox mediator on unbiased conductive substrate is possible if the sampled area is larger than the size of the microelectrode. ${ }^{78}$ For negative feedback, the normalized experimental current is close to the one obtained by simulation for $h_{\mathrm{P}} \approx 0$. For approaching to a conducting surface, the experimental current is slightly lower than that predicted by simulation probably because of kinetic limitations at the carbon microelectrode and/or the unbiased gold substrate.

After mechanically touching the substrate, the current assumes an almost constant value because the probe angle and bending and hence $d$ is not drastically altered upon further movement of the vertical positioning system (eq 1). Figure 4 demonstrates the higher immunity against topographic artifacts of the new soft probes when compared to the previously reported soft stylus probe, mainly due to the preset probe slope and the thin Parylene coating. ${ }^{72}$ The approach curves in Figure 4 also show slightly varying currents at the point of mechanical contact most likely indicating small different probe-substrate distances among the microelectrodes contained in the array. This can for instance be caused by roughness at the cutting line and variation in the carbon track thickness.

High throughput SECM Imaging. SECM imaging in contact regime has been introduced elsewhere. ${ }^{72,75}$ The polymeric probe is pressed against the sample and dragged over the sample while brushing it. The main advantage of this mode is the possibility to scan at constant $d$ over corrugated and tilted surfaces without artifacts and without the need for an instrumentally demanding electronic feedback system to keep $d$ constant. Indeed, as the bended probe can adjust to the sample corrugation and tilt, the probe-substrate distance remains almost constant upon scanning. Consequently, sample leveling before SECM imaging is not needed, even for large area samples. Figure 5 shows a typical 3D SECM image of an interdigitated array of gold microelectrodes shown in Figure $5 \mathrm{~d}$ and obtained with an array of eight carbon microelectrodes ( $250 \mu \mathrm{m}$ interelectrode distance). This test sample was chosen, as it allows the easy detection of a potential

(78) Wipf, D. O.; Bard, A. J. J. Electrochem. Soc. 1991, 138, 469. 


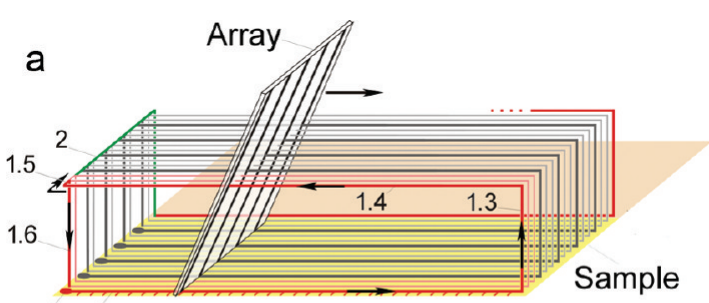

1.11 .2

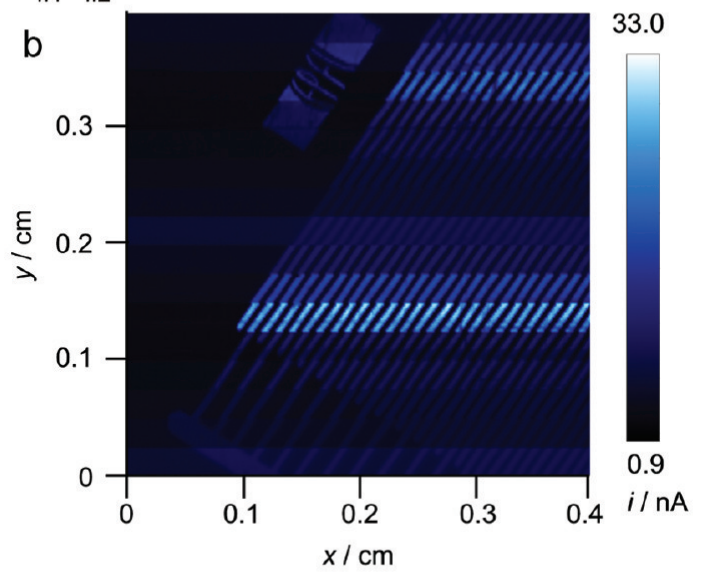

c

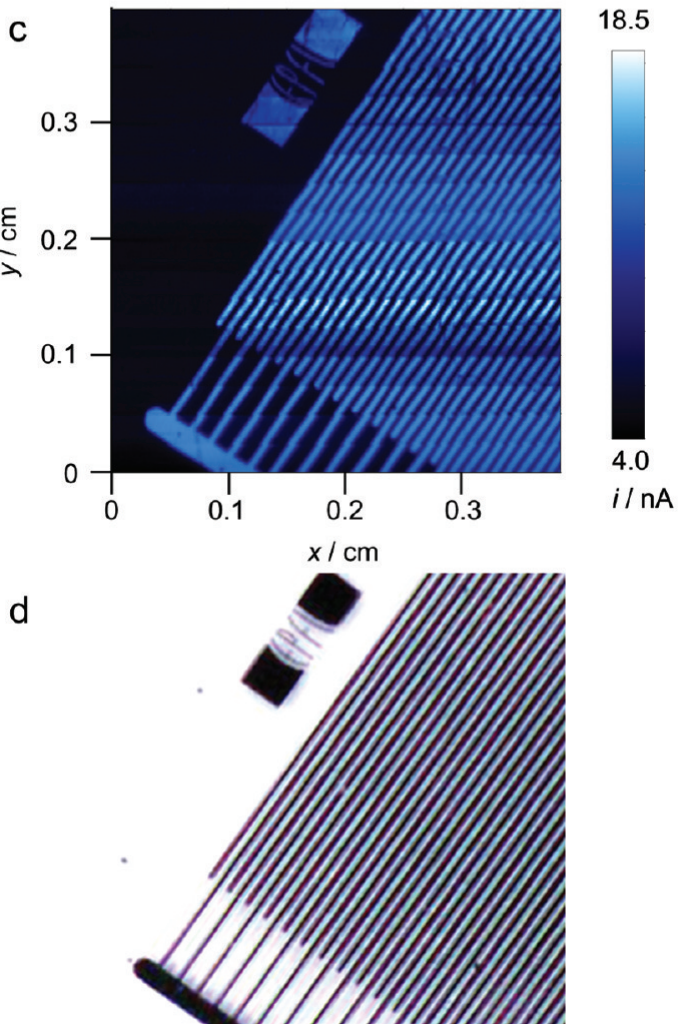

Figure 5. Principle of SECM imaging with a soft microelectrode array. (a) Schematic of the probe array movement (linear multiprobe with five sensors); 1 , line scan of the first sensor; 1.1, starting point; 1.2, forward scan of high-frequency axis; 1.3 , lift off; 1.4, reverse scan; 1.5 , step of standard size of low-frequency axis; 1.6 , reapproach; 2 , large step of low-frequency axis. Black arrows depict the probe scanning direction. (b) SECM feedback image of a gold on glass microstructure with an array of eight microelectrodes and an interelectrode spacing of $250 \mu \mathrm{m}$. $h_{\mathrm{P}}=-60 \mu \mathrm{m}$, step size $=10 \mu \mathrm{m}$ and translation rate $v_{\mathrm{T}}=25 \mu \mathrm{m} / \mathrm{s}$. $2 \mathrm{mM} \mathrm{FcCH} \mathrm{CH}_{2} \mathrm{O}$ with $0.1 \mathrm{M} \mathrm{KCl}$. (c) Image corrected for different sensitivities of the individual microelectrodes and positional offsets of the individual electrodes (details in the text). (d) Optical picture of a gold on glass microstructure employed for this experiment. registration shift and represents at the same time an ideal specimen for proving the soft microelectrode array capacities for surface activity characterization at micrometer resolution over a large area. The image in Figure $5 b$ was constructed from the forward scans data recorded in contact mode. During the reverse scans, the probe is lifted $200 \mu \mathrm{m}$ off the substrate and returned to the starting position with an increment on the low-frequency axis (Figure 5a). Before the next forward scan starts, the probe is lowered by $200 \mu \mathrm{m}$ to re-establish the mechanical contact to the sample. In order to achieve large imaging, the increments are performed 9 times at $25 \mu \mathrm{m}$ steps to completely cover the area between the initial $y$ positions of the microelectrodes. Next, the electrode array is translated by $2000 \mu \mathrm{m}$ to attach a complete new image directly adjacent to the first image. The schematic of this procedure is shown in Figure 5a.

Figure $5 \mathrm{~b}$ shows the uncorrected image. The gold bands are clearly resolved, due to the current contrast originated from the negative and positive feedback achieved over the insulating glass and the unbiased gold bands, respectively. The gold bandwidth and spacing in between gold patterns is equal to $50 \mu \mathrm{m}$, which represents a very high resolution if one takes into account the dimension of the complete area scanned $(0.4 \mathrm{~cm} \times 0.4 \mathrm{~cm})$. This was covered by a total of 401 data points in $x$ direction and a total of 160 individual line scans performed by the eight microelectrodes and defining the point number in $y$ direction. The time required for recording this image with the eight-channel microelectrode array was around $3 \mathrm{~h} 10 \mathrm{~min}$, approximately six times less than the expected time for imaging the same sample region by a single microelectrode and using the same experimental parameters. The deviation from factor eight is due to the lift-off mode that results in additional movements of the positioning system in the $z$ direction and due to a slightly longer data acquisition time that is needed to measure eight current values at each step using the described IVIUM compactstat and the WE32 unit. No particular alignment procedure of sample plane and $x, y$-plane of the positioning system was used.

Figure $5 \mathrm{c}$ shows the corrected image. The corrections include the adjustment of the lateral positions of each individual electrode of the array. While the spacing in the $y$ direction is well-defined by the laser ablation process, the exact position in the $x$ directions is slightly different for each electrode due to the slight misalignment and the cutting procedure with a scalpel. These positional offsets are obvious from the offsets in the image of the straight gold lines in Figure 5b. For correcting the $x$ position of each data point, an offset $x_{\mathrm{offs}, i}$ of the particular electrode that acquired this data point was added to the $x$ position of each point recorded by this electrode. The vector of the correction values is $\mathbf{x}_{\text {offs }} / \mu \mathrm{m}=\{0 ; 5 ; 10 ; 15 ; 20 ; 25 ; 30 ; 35\}$. The resulting data set is no longer an orthogonal grid with equal intervals within one horizontal dimension. In order to plot the image, the data were triangulated by the Delaunay triangulation implemented in the IDL programming language used to produce the image processing software MIRA. After the triangulation was established with 64160 points and 381160 triangles, inner points were interpolated by bilinear interpolation. In addition the measured current values were corrected. First, a current offset $i_{\mathrm{T}, \text { offset }, i}$ was subtracted from each measured current value that reflects an offset between the channels of the microelectrodes, as 
observed in Figure $2 \mathrm{~b}$ at the beginning of each cyclic voltammogram. Such offsets result from a combination of instrumental limitations in the WE32 unit. The different sizes of the individual microelectrode arrays were corrected by a dimensionless scale factor $s_{\mathrm{i}}$ for each microelectrode of the array. Thus, the corrected current value is

$$
i_{\mathrm{T}}{ }^{\prime}=\left(i_{\mathrm{T}}-i_{\mathrm{T}, \text { offset }, i}\right) \times s_{i}
$$

The used values are $\mathbf{i}_{\mathrm{T}, \text { offset }} / \mathrm{nA}=\{3.2 ; 1.5 ; 0.4 ;-1.9 ;-4.0$; $-11.8 ;-4.8 ; 0.1\}$ and $\mathbf{s}=\{3.0 ; 4.0 ; 2.7 ; 1.4 ; 1 ; 0.42 ; 0.72 ; 1.72\}$. The EPFL logo (gold on glass) is resolved, as it can be compared with the optical picture of this sample shown in Figure $5 \mathrm{~d}$. While samples with regular and known patterns such as that in Figure 5 make it easy to select the suitable positional and current correction values, such regions for current and positional referencing can rather easily be incorporated into material libraries. Often an inert background can already serve as a reference point for either the current scaling or offset. However, the array used for Figure 5 represents a rather extreme case of deviation in the response of individual electrodes, and there are probes that do not require current or positional offset corrections at all.

\section{CONCLUSIONS}

The present report illustrates the possibility of performing highthroughput SECM imaging with a linear array of eight microelectrodes prepared by microfabrication techniques. Soft microelectrode arrays are intended to be used in contact regime, avoiding topographic artifacts arising from corrugated or tilt substrates when scanning large sample areas. The surface of the active electrode area can be easily renewed by simple blade cutting, on account of the mechanical stability of the polymeric materials employed for the probe preparation. As a consequence, the array of microelectrodes is capable of being used several times and it could be implemented with minor changes in any conventional SECM setup. The arrangement of the electrodes in a sheet-like body reduces the diffusional interference between them compared to their integration into a planar chip. A drastic reduction of imaging time was achieved for image frames with areas in the $\mathrm{cm}^{2}$ range, while conserving the high sensitivity and resolution of SECM feedback images.

Furthermore, arrays with only eight microelectrodes have been developed so far. We currently work to further automate the probe fabrication and to improve the connection to the potentiostat. With the current equipment, 32 individual electrodes could be used in parallel. This could be increased to 128 by extension with commercially available hardware components, resulting in a linear array able to cover in only one scan a $2.5 \mathrm{~cm}$ wide area with a 200 $\mu$ m resolution (i.e., for a $200 \mu$ m electrode spacing). Imaging with an array of 32 probe electrodes measurements like human fingerprints could be recorded in a few hours. These perspectives offer further possibilities for scanning electrophoresis gels, thin layer chromatographic plates, and material libraries, checking the integrity of corrosion protection coatings, etc.

\section{ACKNOWLEDGMENT}

The Fonds National Suisse pour la Recherche Scientifique (grant no. 20PA21_121570/1 "High throughput SECM imaging") and Deutsche Forschungsgemeinschaft (Wi 1617/10) are thanked for financial support. The technical assistance by Valérie Devaud and Cyrille Hibert is also acknowledged. D.M. and A.L. made an equivalent contribution to this paper.

\section{SUPPORTING INFORMATION AVAILABLE}

Details of probe geometry, mounting, Parylene coating, and working distance of the soft microelectrode array, as well as information about FEM simulations. This material is available free of charge via the Internet at http://pubs.acs.org.

Received for review July 21, 2010. Accepted October 25, 2010.

AC1019304 